\title{
Effect of dose rate on ion beam mixing in $\mathrm{Nb}-\mathrm{Si}$
}

Thomas Banwell and M-A. Nicolet

California Institute of Technology, Pasadena, California 91125

R. S. Averback and L. J. Thompson

Materials Science and Technology Division, Argonne National Laboratory, Argonne, Illinois 60439

(Received 10 January 1986; accepted for publication 8 April 1986)

The influence of dose rate, i.e., ion flux, on ion beam mixing in Nb-Si bilayer samples was measured at room temperature and $325^{\circ} \mathrm{C}$. At the higher temperature, an increase in dose rate of a factor of 20 caused a decrease in the thickness of the mixed layer by a factor of 1.6 for equal total doses. At room temperature, the same change in flux had no effect on mixing. These results are consistent with radiation-enhanced diffusion theory in the recombination-limited regime.

The temperature dependence of ion beam mixing (IM) has been investigated in many systems, ${ }^{\prime}$ and it has been observed that mixing is usually independent of temperature at low temperatures and dependent on temperature at high temperatures. Most fundamental studies of IM have focused on the low-temperature regime. There are, however, many unanswered questions regarding IM in the high-temperature regime. In fact, the underlying reason(s) for the temperature dependence at high temperatures is still not firmly established. It is generally believed that the temperature dependence is a consequence of radiation-enhanced diffusion (RED). However, RED can give rise to a temperature dependence by various mechanisms: temperature-dependent defect production, a temperature-dependent sink structure, or recombination of migrating point defects, i.e., vacancies and interstitials. ${ }^{2,3}$ Diffusion during a thermal spike can theoretically account for the temperature dependence in some systems. ${ }^{4.5}$ Additional insight regarding the mechanisms of high-temperature mixing can be obtained by measuring the influence of dose rate on IM in conjunction with the temperature dependence. This combination of measurements is particularly illuminating as it discriminates between some of the proposed mechanisms for high-temperature IM. Specifically, if recombination of migrating point defects is the underlying cause of the temperature dependence in IM, there must be a corresponding dose rate dependence. No influence of dose rate is expected for the other mechanisms.

Previous investigations of dose rate effects in IM have been inconclusive. In one case, mixing in a $\mathrm{Ni}$-Si bilayer sample was studied, and no effect of dose rate was observed. "In $\mathrm{Ni}-\mathrm{Si}$, however, the temperature dependence is much weaker than one would expect from RED in the recombination regime." In another study, using a metal "marker" geometry ( an Au marker in $\mathrm{Ni}$ ), a small influence of dose rate on IM was observed. ${ }^{7}$ However, a simple interpretation of this experiment based on RED was not possible as at the higher temperatures the temperature dependence grew stronger but the dose rate effect disappeared. In the present letter, the effect of dose rate on IM in a Nb-Si bilayer sample is reported. Nb-Si was selected for this investigation as the temperature dependence of IM in this system was previously determined and is strong, unlike $\mathrm{Ni}-\mathrm{Si}^{8}{ }^{8}$

The theory for RED and the associated effect of dose rate is well developed, ${ }^{2,3}$ and has recently been applied to IM. ${ }^{6}$ Before outlining the results of this theory, it is helpful to consider the underlying basis for a dose rate effect. The fundamental basis for a dose rate effect is that the changes in the system produced by an incident ion depend on the state of the system prior to the impact, that is, there is a correlation between individual impact events. If the system relaxes in a time $\tau$ after an impact, and the altered region associated with this impact event extends over a projected area $A$, then the probability for one or more overlapping events within $\tau$ is $1-\exp (-\tau A \dot{\Phi})$, where $\dot{\Phi}$ is the incident particle flux. A dose rate effect exists if, and only if (i) events are correlated and (ii) $\tau A \dot{\Phi}>1$. For an intracascade process (e.g., thermal spike), condition (ii) requires currents exceeding $2000 \mathrm{~A} /$ $\mathrm{cm}^{2}$. With RED, lifetimes are long enough such that (ii) is readily satisfied. If a constant density of fixed sinks annihilate defects responsible for atomic diffusion, then there is no correlation between these defects and no dose rate effect can occur. If defects interact with one another (e.g., form complexes, recombine, etc.), then there is correlation and a dose rate effect.

In the recombination regime of RED, the dominant defect reaction is the recombination of vacancies and interstitials. Therefore, the diffusion length of each defect, and consequently the mixing per radiation-induced defect, or unit dose, is determined by the instantaneous concentration of defects. The concentration of defects depends on the time necessary for the slowest moving defect species to find stationary sinks and the rate at which defects are generated. ${ }^{3}$ As the mobility of the defects depends on temperature and the defect generation on dose rate, IM will depend on both of these variables in this recombination-limited regime.

The growth of $\mathrm{NbSi}_{2}$ follows nearly

$$
\frac{d x^{2}}{d \Phi}=2 \Omega \Delta C \frac{D}{\dot{\Phi}},
$$

where $x$ is the thickness of $\mathrm{NbSi}_{2}$ formed; $\Phi, \dot{\Phi}$ are dose and dose rate, respectively. $\Delta C$ is the difference in concentration of the moving species across the mixed layer and $\Omega$ is the atomic volume of $\mathrm{NbSi}_{2}$. D is the atomic diffusivity within the mixed region. Sizmann ${ }^{3}$ has reviewed the behavior expected for simple defect interactions in RED. This work suggests that at quasi-equilibrium

$$
D \sim D_{0}(T) \dot{\Phi}^{\beta},
$$

where $1 / 2 \leqslant \beta \leqslant 1$. There are two significant limiting cases. Case (a) $\beta \rightarrow 1 / 2$ when binary recombination dominates, 
and $D_{v} \propto \exp \left(-H_{m}^{v} / 2 k T\right)$ where $H_{m}^{v}$ is the migration enthalpy for vacancies. We assume here that vacancies are less mobile than interstitials. $T$ is the irradiation temperature. Case (b) $\beta \rightarrow 1$ when defect annihilation at sinks dominates, and $D_{0}$ is only weakly $T$ dependent.

A useful approximation to consider is therefore

$$
\frac{d x^{2}}{d \Phi} \sim 2 \Omega \Delta C D_{0}(T) \dot{\Phi}^{\beta-1}
$$

For case (b), the mixing is independent of both temperature and dose rate, whereas case (a) depends on both.

Highly polished Al plates were used as supporting substrates in this study. $150 \mathrm{~nm} \mathrm{Si}, 45 \mathrm{~nm} \mathrm{Nb}$, and $10 \mathrm{~nm} \mathrm{Si} \mathrm{films}$ were sequentially evaporated onto the $\mathrm{Al}$ substrates in an oilfree $e$-beam system. The $10 \mathrm{~nm} \mathrm{Si} \mathrm{cap} \mathrm{was} \mathrm{used} \mathrm{to} \mathrm{inhibit}$ oxidation, especially at elevated temperatures. The Al plates were fastened to an implantation carousel of large thermal mass. Irradiations were performed with $225 \mathrm{keV}$ Ar to prescribed fluences in the range $4-30 \times 10^{15} \mathrm{Ar} \mathrm{cm}^{-2}$. The measured sample temperature was held at either $24 \pm 2{ }^{\circ} \mathrm{C}$ or $329 \pm 2{ }^{\circ} \mathrm{C}$ during irradiation. The incident Ar beam was rastered over the sample. The average beam current was set at either $0.05 \mu \mathrm{A} / \mathrm{cm}^{2}$ or $1.0 \mu \mathrm{A} / \mathrm{cm}^{2}$, giving a factor of twenty variation. Irradiations at high and low currents were alternated to minimize correlations with long term temperature drifts. After the irradiations were completed, the specimens were cooled to room temperature and analyzed by 1.5 $\mathrm{MeV}$ He backscattering spectrometry. Subsequent to analysis, the specimens were reheated to the irradiation temperature and annealed for several hours to test for thermally induced growth of the mixed layer. No additional mixing was observed during this annealing treatment.

In Fig. 1, backscattering spectra clearly show the effect of a difference of 20 in dose rate on IM at $325^{\circ} \mathrm{C}$. It can be observed in both spectra that the mixed layer has a rather constant composition, corresponding to $\mathrm{NbSi}_{2}$. The $\mathrm{NbSi}_{2}$ phase was identified in previous studies of both mixing and thermal reactions. ${ }^{9.16}$ The thicknesses of mixed layer $x$ are plotted as a function of the square root of total dose $\Phi$ in Fig. 2 ; results for two temperatures and two dose rates are illustrated. The data at $325^{\circ} \mathrm{C}$ seem to indicate that there is an incubation period, during which the mixing rate is slow and there is little dose rate effect. At higher doses, the mixing rate increases, approaches a square root of dose dependence, and is dose rate dependent. Using the slopes of the mixing versus square root of dose curves at the higher doses as the basis for comparing mixing for the two dose rates, it is found that the ratio of the mixing rates for low and high dose rates is a factor of 1.6. At the lower temperature, the dose dependence of mixing was determined for only one dose rate. For it, a square root of dose dependence is observed with no evidence of an incubation dose. For the one measurement at the reduced dose rate, the mixing rate is unchanged, although we cannot be certain that a square root of dose dependence holds at the lower dose rate as it does for the higher.

The results of these experiments are in qualitative agreement with the RED model of mixing outlined above, i.e., there is a dose rate dependence in the temperature dependent (high temperature) regime, and no dependence on dose rate

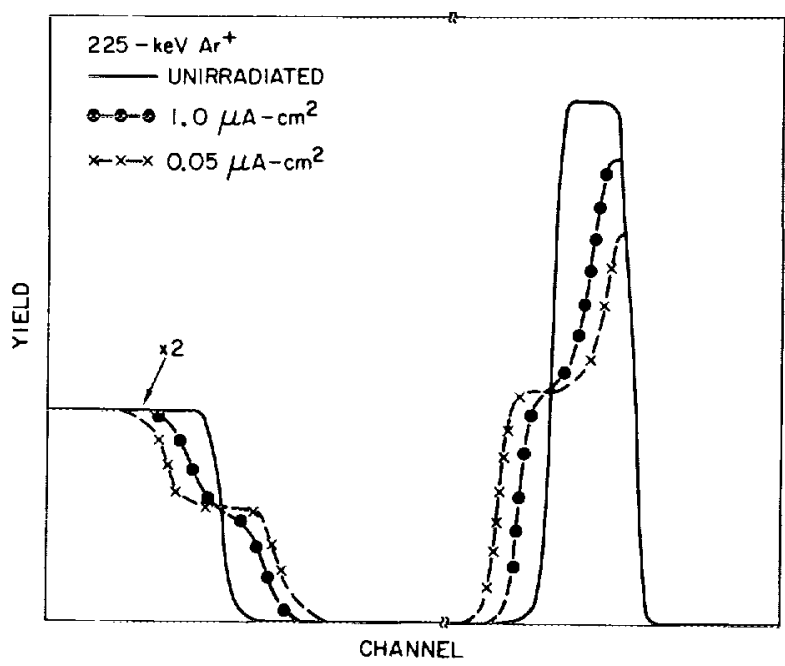

FIG. 1. Backscattering spectra showing the effect of dose rate on ion beam mixing. The specimens were both irradiated at $325^{\circ} \mathrm{C}$ with $225 \mathrm{keV} \mathrm{Ar}$, but at different dose rates. (The signals from the $10 \mathrm{~nm}$ Si caps have been removed from the spectra for clarity.)

in the temperature independent (low temperature) regime. We deduce from the factor of 1.6 reduction in mixing rate for a factor of 20 increase in Ar current that $\beta=0.66$ using Eq. (3). This is consistent with a RED model in which recombination dominates although annihilation at sinks is not negligible. Several interferences can alter the apparent value of $\beta$, including assumptions of steady state and beam heating effects. Some aspects of mixing, however, are not predicted by the model. The model does not predict an incubation period, i.e., it predicts a strictly square root of dose dependence. The model leading to Eq. (3) assumes that the specimen is in steady state from the outset of the irradiation, even though a mixed $\left(\mathrm{NbSi}_{2}\right)$ layer has not yet formed. Therefore, it is possible that an incubation dose is necessary to establish steady state conditions. Beam heating is a possible source of error as the high dose rates would tend to raise the specimen temperature, and thereby increase the mixing rate. Using the temperature dependence of mixing in $\mathrm{Nb}-\mathrm{Si}$ at $325^{\circ} \mathrm{C}$ reported by Matteson et al., ${ }^{9}$ it is found that the specimen would

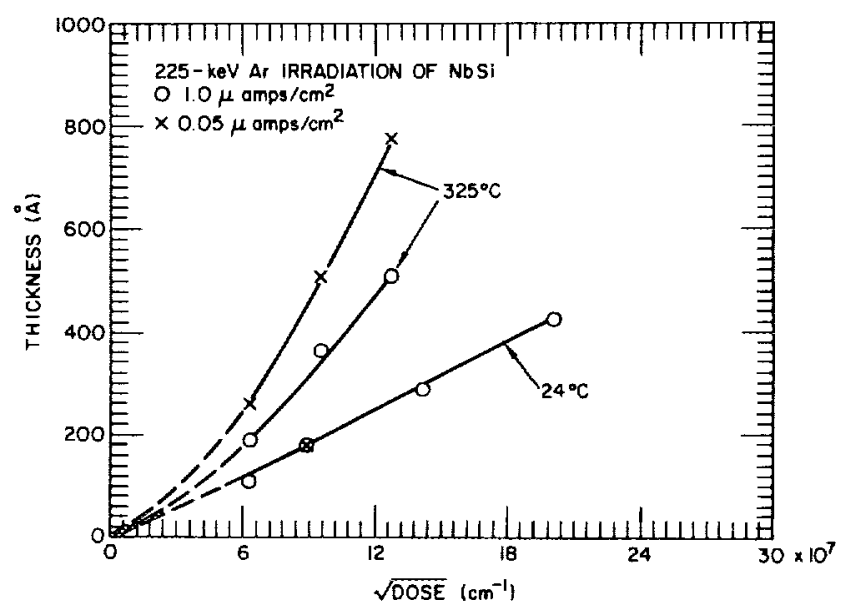

FIG. 2. Thicknesses of mixed layers in Nb-Si bilayer specimens as a function of the square root of dose for two dose rates and two temperatures. 
have had to have increased in temperature by $20^{\circ} \mathrm{C}$ to change the apparent power law $\beta$ from 0.5 to 0.66 . This is much greater than was calculated for the irradiation conditions employed here. Ion mixing is independent of temperature at $24^{\circ} \mathrm{C},{ }^{9}$ consequently beam heating effects at $24^{\circ} \mathrm{C}$ are much less significant than at $325^{\circ} \mathrm{C}$. Another possible source of error is that the beam was rastered at $1 \mathrm{kHz}$ and not locally continuous. As the rastering was kept constant for the two dose rates and only the peak intensities changed, the basic effect that we report here must be a consequence of the dose rate. Moreover, we do not believe that rastering strongly influences the kinetics of IM in the present case. The instantaneous vacancy concentrations in our specimens, calculated for the irradiation conditions at $325^{\circ} \mathrm{C}$, are $10^{-2}-10^{-3} .^{3}$ As the concentrations of vacancies produced in each sweep are $10^{-5}-10^{-6}$, no significant relaxation occurs between sweeps. Finally we note that some of the assumptions, such as a completely homogeneous specimen, are extreme.

It may be expected that at low temperatures, when the defects are slowly moving, the defect concentration would eventually become larger than the sink concentration and that case (a) would apply. Moreover, at high temperatures, the defects are very mobile and correspondingly, it may be expected that fixed sinks would become the main source for defect annihilation. This is, of course, opposite to the experimental results reported here and elsewhere. A plausible reason for this behavior is that the sinks are not fixed sinks existing in the specimen prior to irradiation, but rather are (quasi-) sinks formed by the clustering of defects during low-temperature irradiation. The concentration of these clusters becomes so large during low-temperature irradiation that all newly produced defects lie within the reaction radii of the clusters and no long range migration can occur.
Until these clusters can migrate or dissolve at higher temperatures, defect reactions with these clusters will dominate and suppress RED.

The results of these experiments demonstrate that standard RED theory involving the recombination of radiationinduced point defects is adequate to explain the temperature dependence of IM in $\mathrm{Nb}-\mathrm{Si}$. The reported activation enthalpy for the diffusion coefficient during mixing, $0.9 \mathrm{eV}$, is a reasonable value of $H_{m}^{\prime \prime} / 2$. This experiment proves that thermal spike mixing is not the dominant high-temperature process in $\mathrm{Nb}-\mathrm{Si}$, contrary to previous speculation. ${ }^{+}$The mechanism for the temperature dependence of mixing in $\mathrm{Ni}$ $\mathrm{Si}$ and $\mathrm{Cr}$-Si remains an open question.

This work was supported at Argonne National Laboratory by the U.S. Department of Energy, BES-Materials Sciences, under contract No. W-31-109-Eng-38. Partial financial support at the California Institute of Technology provided by the Office of Naval Research under contract No. N00014-84-16-0275 is also acknowledged.

'See, e.g., B. Paine and R. S. Averback, Nucl. Instrum. Methods B 7/8, 666 (1985).

${ }^{2}$ G. J. Dienes and A. C. Damask, J. Appl. Phys. 29, 1713 (1958).

'R. Sizmann, J. Nucl. Mater. 69/70, 386 (1968).

${ }^{4}$ U. Shreter, F. C. T. So, B. M. Paine, and M-A. Nicolet, Materials Research Society Symposium Proceedings, Vol. 27 (Elsevier, New York, 1984), p. 31.

'D. Peak and R. S. A verback, Nucl. Instrum. Methods B 7/8, 561 (1985).

${ }^{\circ}$ R. S. A verback, L. J. Thompson, J. Moyle, and M. Schalit, J. Appl. Phys. 53, 1342 (1982)

'J. Bottiger, S. K. Nielsen, and P. T. Thorsen, J. Phys. (Paris) (in press). ${ }^{9}$ L. S. Wielunski, B. M. Paine, B. X. Liu, C.-D. Lien, and M-A. Nicolet, Phys. Status Solidi A 72, 399 (1982).

${ }^{9} \mathrm{~S}$. Matteson, J. Roth, and M-A. Nicolet, Radiat. Eff. 42, 217 (1979).

'T. Kanayama, H. Tanoue, and T. Tsurushima, Appl. Phys. Lett. 35, 222 (1979) 